Volume 10, No.1, January - February 2021

International Journal of Advanced Trends in Computer Science and Engineering

Available Online at http://www.warse.org/IJATCSE/static/pdf/file/ijatcse301012021.pdf

https://doi.org/10.30534/ijatcse/2021/301012021

\title{
New security approach-based Steganography and Cryptography by modified AES algorithm
}

\author{
Suraj Kumar ${ }^{1}$, Dr. Surender Soni ${ }^{2}$ \\ ${ }^{1}$ National Institute of Technology, Hamirpur, H.P-177005, India, surajk21.in@ gmail.com \\ ${ }^{2}$ National Institute of Technology, Hamirpur, H.P-177005, India, soni@nith.ac.in
}

\begin{abstract}
The transmission of information through any channel of correspondence needs solid encryption procedures with the end goal of information security. The computerized watermarking assumes a significant part in inserting data into an advanced picture signal, for the check and character of its proprietors. In discrete wavelet change, "investigation channel bank" can be utilized for dissecting picture signals by going through. The channel bank comprises high and low pass channels at every deterioration stage. In this paper, a technique to consolidate steganography (Least Significant Method-LSM) and cryptography Advanced Encryption Standard (AES) is thought to give a safer method to information transmission through any unstable or public organizations. Prior to inserting the content in the picture, the text is scrambled utilizing AES calculation. Utilizing least significant bit (LSB) strategy, encoded the text installed in low-low (LL) subband wavelet decayed of a picture. Converse wavelet changes applied will lead to the output picture is communicated to the recipient. Presently at the collector's end, the picture changed utilizing wavelet and encoded text will be extricated by utilizing the LSB technique. This paper shows how AES calculation is utilized in the decoding of the outcome.
\end{abstract}

Key words : AES, Encryption, Transmission, watermarking, wavelet.

\section{INTRODUCTION}

Transmission of information through any channel of correspondence needs data security is a fundamental worry for all the independent clients of an organization. Swanson, kobayashi, \& Tewfik [1] explain how advancements in straightforward information installing and watermarking for sound, picture, and video use for securing message. The current day programmers will be danger for information and danger hangs like Damocles blade. Thien \& Lin [2] tell about the investigation presents a basic technique for high-concealing limit. The essential idea utilizes a modulus activity. Transmitting of information through some channel of correspondence needs solid encryption methods with the end goal of information security. Wang, Lin, \& Lin [3] build up a strategy to insert significant information in the host picture so the interceptors won't see about the presence of the information. V.Kartalopoulos [4] explain why data security is significant, what is being done about it, how it applies to networks, and an outline of its central points of interest. New patterns and improvements in data innovation feature the requirement for protection, secure to the ensured transmission of information. Regular encryption strategies neglected to the obtain ideal consequence of ensuring information. The straightforward route is to keep coming up for passwords and remarkable id and blend of letter sets and mathematical. AES has arisen as a leader and productive calculation due to natural inbuilt in a preferred position of better security with minimum usage intricacy. Broad examination in the picture coding for picture pressure applications, DWT (Discrete wavelet transform) fills in a standard instrument, for their information decrease capacity. The total picture is packed, changed into a solitary information object by the wavelet pressure framework, as opposed to impede by block as in the discrete cosine transform (DCT) based pressure framework. At some point when the whole picture is accomplished, there will be a uniform dispersion of pressure blunder across that picture. A picture goal improvement in wavelet space is a matter of concern for additional examination and as of late numerous new calculations have been suggested. DWT is the most suitable applications. Discrete wavelets transform decays a picture into various subband pictures. They are called by high-high, high-low, low-high and low-low. Here subgroups have a similar size as the information picture. Zeng, X.-t.."s strategy depends on the number wavelet change to improve the inserting limit [5].

\section{METHODOLOGY}

In this paper, another technique is utilized to give information in more of a made sure way. One of the symmetric key methods encodes content to be transmitted: AES with a key. Under this cycle by utilizing a key, the given content is encoded. At that point, this resultant content is unscrambled with a similar key (the key equal to size 56-cycle). For Obtaining figure text implanted in the LL sub-band of wavelet changed the picture. The strategy to install the information is the LSM. In Process-1, this approach is depicted. Jung \& Yoo 
[6] clarify why Least-critical piece (LSB) replacement is one of the renowned procedures applied in steganography, which makes adjustments to the cover picture by essentially subbing mystery bits for the LSBs of the cover pixel. Notice that, as the LSB is being modified (no, or \pm 1 change to given pixel esteem). Since the natural eye can't discover a distinction between the first picture and the watermarked picture. Whereas Chang \& Cheng [7] explain that how by applying an ideal pixel change cycle to the stego-picture got by the straightforward LSB replacement technique, the picture nature of the stego-picture can be significantly improved with low extra computational unpredictability. A modification to the Least Significantit bit (LSB) coordinating, a steganographic strategy for implanting message bits into an actually picture [8]. In the LSB coordinating the decision of whether to add or deduct one from the cover picture pixel is arbitrary. When the code text is installed in sub-band LL, a backward wavelet change is applied. At that point, the outcome picture shipped off the collector.

\subsection{Process-1: LSM}

- The estimation of the pixel.

- Convert to its identical paired structure.

- Modify the most un-critical piece as needs are.

Recipient end, the beneficiary will forward wavelet the change of picture obtained. By and by, from the LL sub-band, the substance is isolated. The removed content which has a mixed structure is unscrambled utilizing one key. Wavelet-based steganography is having another thought autonomous of the utilization of wavelets. Here the information is taken care of to the extent wavelet coefficient of images. Notwithstanding, in the LSB method some change in a bit of the authentic pixel.

\subsection{Process-2: HAAR WAVELET}

Haar change return numerous coefficient that is 0 or shut to 0 . Kumar \& Reddy [9] explain how a specific sort of pressure procedures is by utilizing wavelet transform. In the wake of taking haar change of a picture, thought to shroud bits in coefficients that underneath has limit esteem. Equivalent being haar converse of the altered information. Hypothetically it has been seen that there ought not to do a lot of change of a picture because that we are concealing of the piece's irrelevant coefficients.

Execution:

ENCRYPT:

- Take wavelet change about a picture.

- Discover the coefficients under edge esteem.

- Supplant these pieces with pieces of information to be covered up.

- Take the opposite change.

- Store as an ordinary picture, in standard form.

\section{DECRYPT:}

- Take wavelet change about a picture.

- Discover the coefficients under edge esteem.

- Concentrate pieces of information from coefficients.

- Join extricated information bits to a genuine message.

\subsection{Process-3: A E S}

Where encryption is a symmetric calculation where we can utilize encryption (one key) and unscrambling the same key. A sender may use and beneficiary. In AES there are 128,192, and 259 pieces in length, every one of them contains 2128 , 2256, and 2192 mixes. The mystery kept up by the key is made sure about and verification is kept up the key itself. In this both the keys should be left well enough alone. However, without not public keys or possibly other data difficult to decipher of code text. With the assistance of not private keys, the calculation should be lacking to locate not public keys. We need mystery and verification, just one key will be sufficient that is a not public key for encryption. In cryptographic arrangements Data Encryption Standard and Advanced Encryption Standard give securities however from cryptographic, one is symmetric, other will have deviated. The advanced Encryption Standard key will be very hard to dismantle than Data Encryption Standard, both need very careful managing out to the appropriate key among the dispatcher and beneficiary. Advanced Encryption Standard calculation defined in Figure 1 underneath.

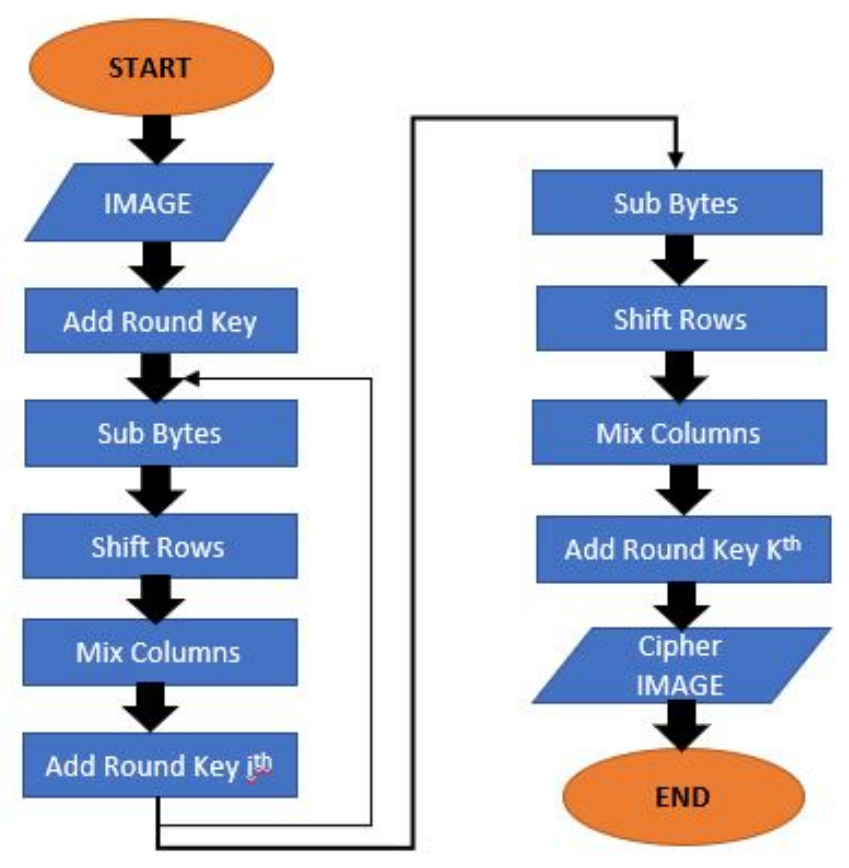

Figure 1: AES algorithm 


\subsection{Modify AES:}

The imperatives of the applications and of equipment utilized put the advancement of calculations existed to limit the computational intricacy, the capacity memory and increment security execution. Since the calculations are worked by numerical changes which treat data as numbers. The augmentation tasks burn-through additional handling times contrasted with different activities. The Mix Column change in AES depends on the duplication of sections. Thus, a change by another cycle of less execution and simultaneously holding the guideline of Shannon in the dispersion and disarray. The proposed calculation was created on the AES with Shift-Columns change or the name Shift-AES. Move AES contains four squares as follows: Shift Cols, Sub Bytes, Shift Rows, and Add Round Key, introduced in Figure 2. The accompanying plan exhibits the best improvement at the degree of entropy and of the execution season of the processor [10].

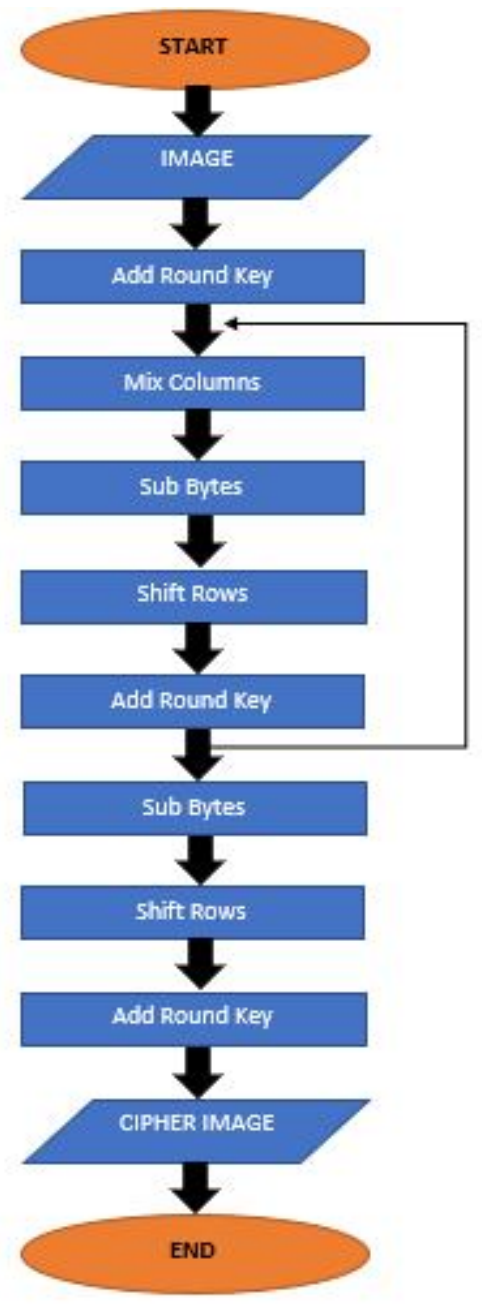

Figure 2: Modified AES algorithm

\subsection{Conversion from Plain Text to Cipher Text}

AES is a square code. It works on plain content with a square of pieces and returns figure text of a similar size. In this, we have performed 10/12/14 rounds. It contains the byte substation, many move lines, blend sections, and afterward add around a key. Substitute of every byte utilizes one table 16 x16 byte, contain change of all determined qualities. Every byte state supplanted by byte filed: line, section. Move lines utilized round about byte. Move-in each first line is unaltered and second column is 1 byte round move to one side, the third line is 2 bytes roundabout move to one side moreover it might measure and decode transforms utilizing roundabout move to the right. In the blend sections, every segment is prepared and isolated and every byte is supplanted by a worth subject to bytes in the segment. Furthermore, A XOR state with 128 key pieces managed by section is around key added and backward for decryption. AES unscrambling isn't indistinguishable from encryption since the means done backward request yet is characterized as identical reverse code with ventures concerning encryption by utilizing converse of each progression with an alternate key.

\subsection{Ordering picture information}

A picture is treated as a two-dimensional exhibit of coefficients, each coefficient addressing the brilliance degree in there. When looking from a higher viewpoint, we can't distinguish between coefficients as more significant ones, and lower significant ones. Be as it could, thinking all the more obviously, we can. Most of ordinary pictures have smooth shading varieties, with the nice subtleties being addressed as sharp edges in the middle of $t$ smooth varieties. In reality, the unwrinkled varieties in shading can be known as low recurrence varieties and wrinkled varieties as high recurrence varieties. The very low recurrence segments (unwrinkled types) comprise the base of a picture, and the high recurrence segments (edges that give the detail) add upon them for refining the picture, like this giving a definite picture. Consequently, the smooth varieties are asking more significance than the subtleties. Isolating the smooth varieties and subtleties of the picture ought to be possible from various viewpoints. One way is that the disintegration of the picture using a Wavelet Transform in Discrete form.

\section{The Discrete wavelet transforms of a picture}

The plan goes this way. The low pass filter (LPF) and a high pass filter (HPF) are chosen, with the end goal, they precisely split the recurrence range between themselves. This channel pair is known as the Analysis Filter pair. To begin with, the very low pass channel is used for every line of data, along these lines getting minimal recurrence segments of the line. But since the low pass filter is a half band channel, and return information includes frequencies just in the primary portion of the initial recurrence range. Along these lines, by Shannon's Sampling Theorem, they can be subsampled by 
two, with the goal that the return information currently contains only huge bit of the main numbers of test . Currently, the hpf is used to get a same types pillar of data, as well as the large pass parts are dispersed, and putting from the side of the low pass sections. The system will accomplished for all column. Then, the separating is accomplished for every single part of halfway informations. The following 2-D exhibit of coefficients comprises of four group of the data, named as HL (high-low), LL (low-low), HH (high-high) and LH (low-high). The low-low band can be weakened by in a similar manner, like this creating many more sub-groups. This ought to be possible upto any amount, along these lines, causing a pyramidal decay as demonstrated in Figure 3 underneath.

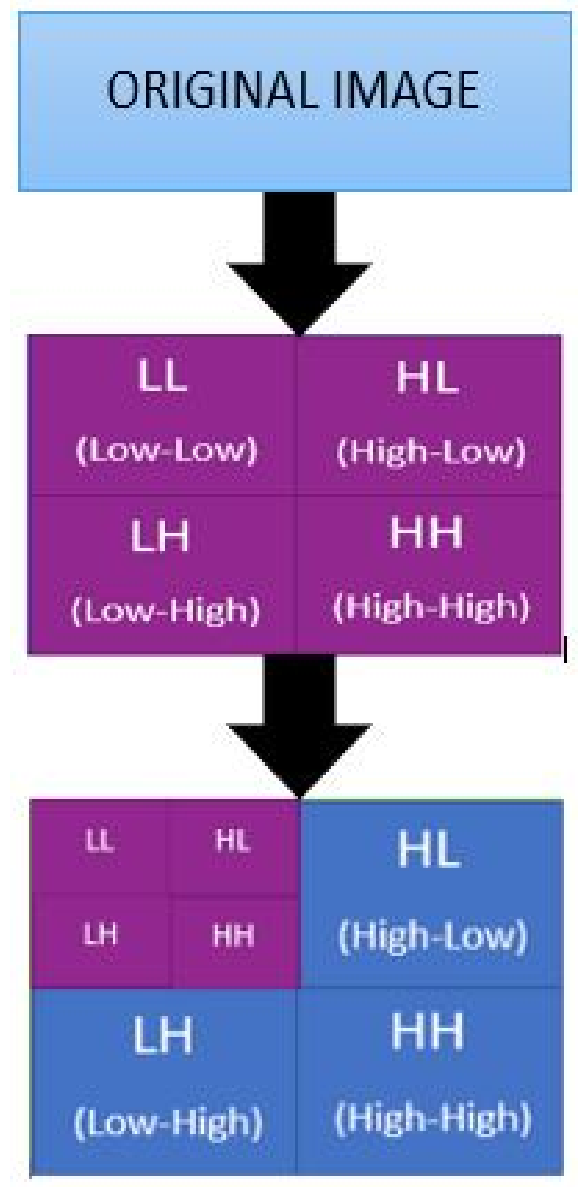

Figure 3: DWT Decomposition

The Inverse Discrete wavelet transform of an image

Likewise, as a forwarded for a switch to isolate an image information into many classes of significance, an opposite shift is reassembling many of the different class of information into a reproduced picture. A couple of high-pass and low-pass stations are used. The channel pair is referred to as the Synthesis Filter pair. A sifting technique is the polar opposite and we begin from a highest degree, use the channels, column-wise, first and afterward row-wise, and the next procedure to the following degree, until we arrive at the principal level.

\section{IMPLEMENTATION}

\subsection{Choosing an Image document}

To begin with, select any picture record, behind which the client needs to shroud information. The picture which is chosen ought to have fixed width and tallness. Presently save picture record as jpeg expansion and a picture shows up as a unique picture document.

\subsection{Picture Steganography}

\section{Sender Side}

will choose the first picture in jpeg expansion design. Presently sender will read documents utilizing ,imread" work. LIAW, CHANG, \& LIAO [11] explain that Steganographic procedures Steganography is the technique for concealing information in such a way that nobody, aside from the sender and the planned beneficiary, anticipates the presence of the shrouded information. where as Radhakrishnan, Kharrazi, \& Memon [12] propose another type of interactive media steganography called information concealing. Also, conversion of picture record from color to black and white, utilizing capacity ,rgb2gray”. After reading content, furthermore, convert texts to the double arrangement. At that point, the key is perused and the content changes over into encoded design. When the wavelet change work, for example, sumdiff() utilized. A picture can isolate into subgroups as LH, LL, HH, HL. A parallel code must be placed in Subband LL by utilizing embeddingfunc() which can be seen in Figure 4. We may apply opposite wavelet change capacity, convert a picture in some unique size, and a picture is shipped to a beneficiary.

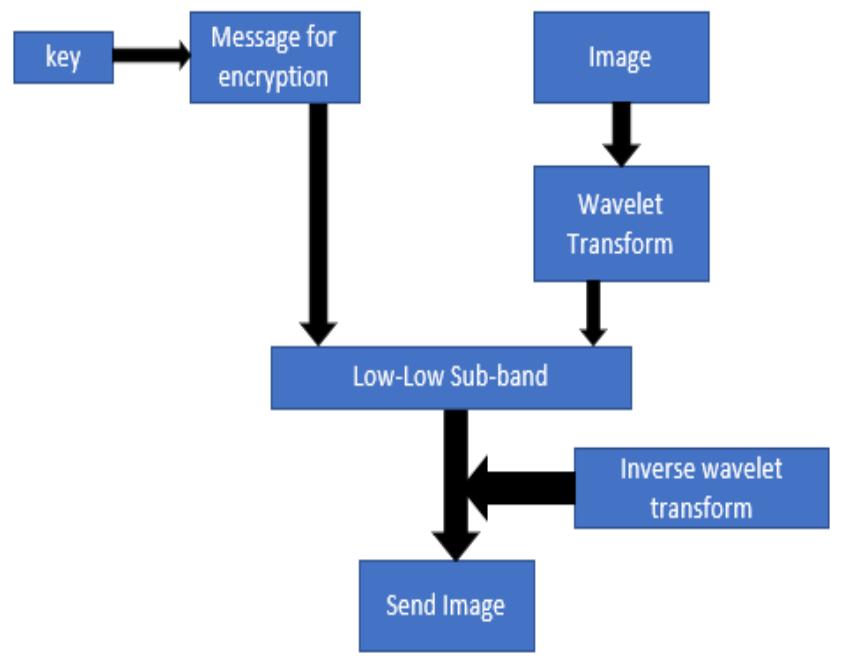

Figure 4: Sender side (image stenography)

\section{Making Stego-Image record}

Making stegno-Image document, join stego-text record, and stego-picture record utilizing advanced watermarking. It will 
give structures of stego-picture text record on a transmitter side in which concealed content available.

\section{Receiver Side}

At the point when the beneficiary peruses the content document utilizing ,fread", it gets changed over into a picture. For a recipient apply wavelet change work sumdiff( ) and partition the picture to four sub-groups as LH, LL, HH, HL. Presently pick up necessary subband LL from a picture. Utilizing extractionfun2 ( ) to remove code from a picture, convert in hexadecimal configuration, afterward stored in a variable ,extra1" which can be seen in Figure 5.After that decode scrambled code utilizing "des1keydecrfunc ( )".

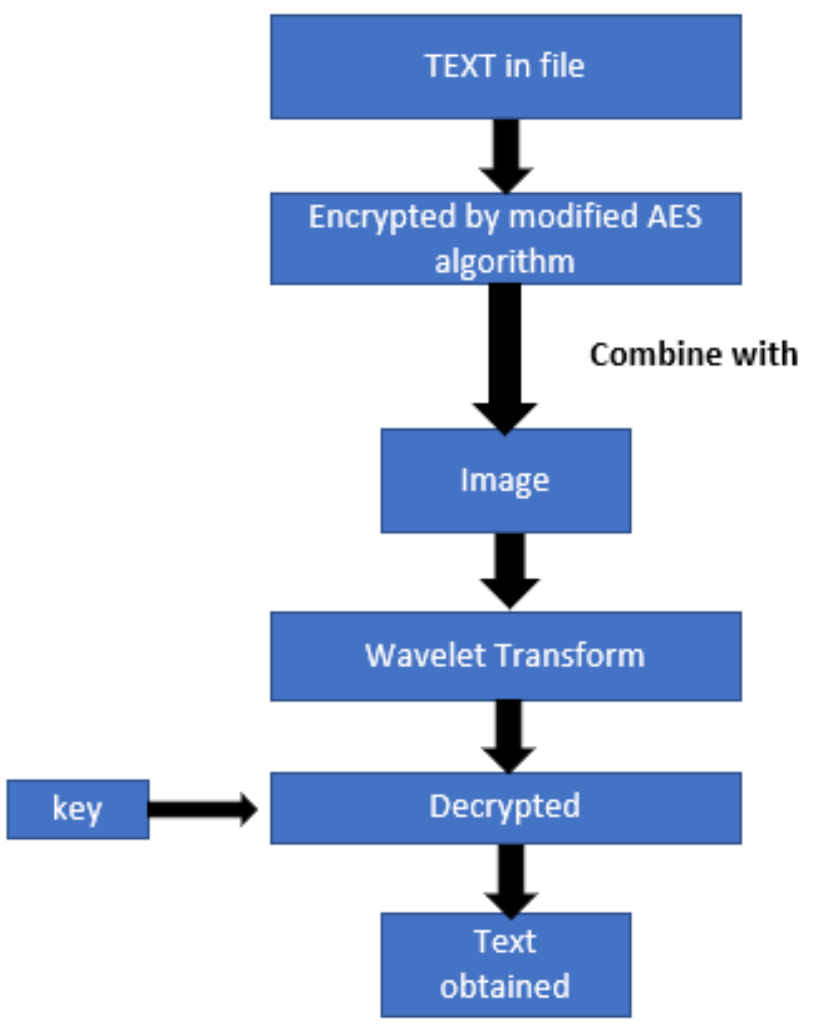

Figure 5: Receiver side (image stenography)

\subsection{Picture Recover}

Picture document is perused by capacity ,imgread" and content record is open utilizing fopen work and to put away in a variable ,fid". Utilizing capacity fread and put away in a variable ,a". Presently converted content document in picture record utilizing network portrayal. Here to play out some expansion and deductions on a network it is set to the appropriate subband ( HH, LL, HL, LH). A picture can be recuperated by content utilizing ,extractionfun".

\subsection{Principle Diagram of proposed Model:}

In proposed engineering, implanted data is taken as text. Prior to inserting content in a picture, text encoded utilizing AES calculation. Content may be a sentence or a key with a length of 8 characters in alphabetic words. Utilizing LSB strategy, scrambled content was inserted in the "LL sub-band wavelet decayed picture". Converse wavelet change will be applied and a resultant picture is sent to a collector. Presently at a recipient's end, a picture changed utilizing wavelet, scrambled content extricated by utilizing at least a significant bit (LSB) technique which is shown by Figure 6.

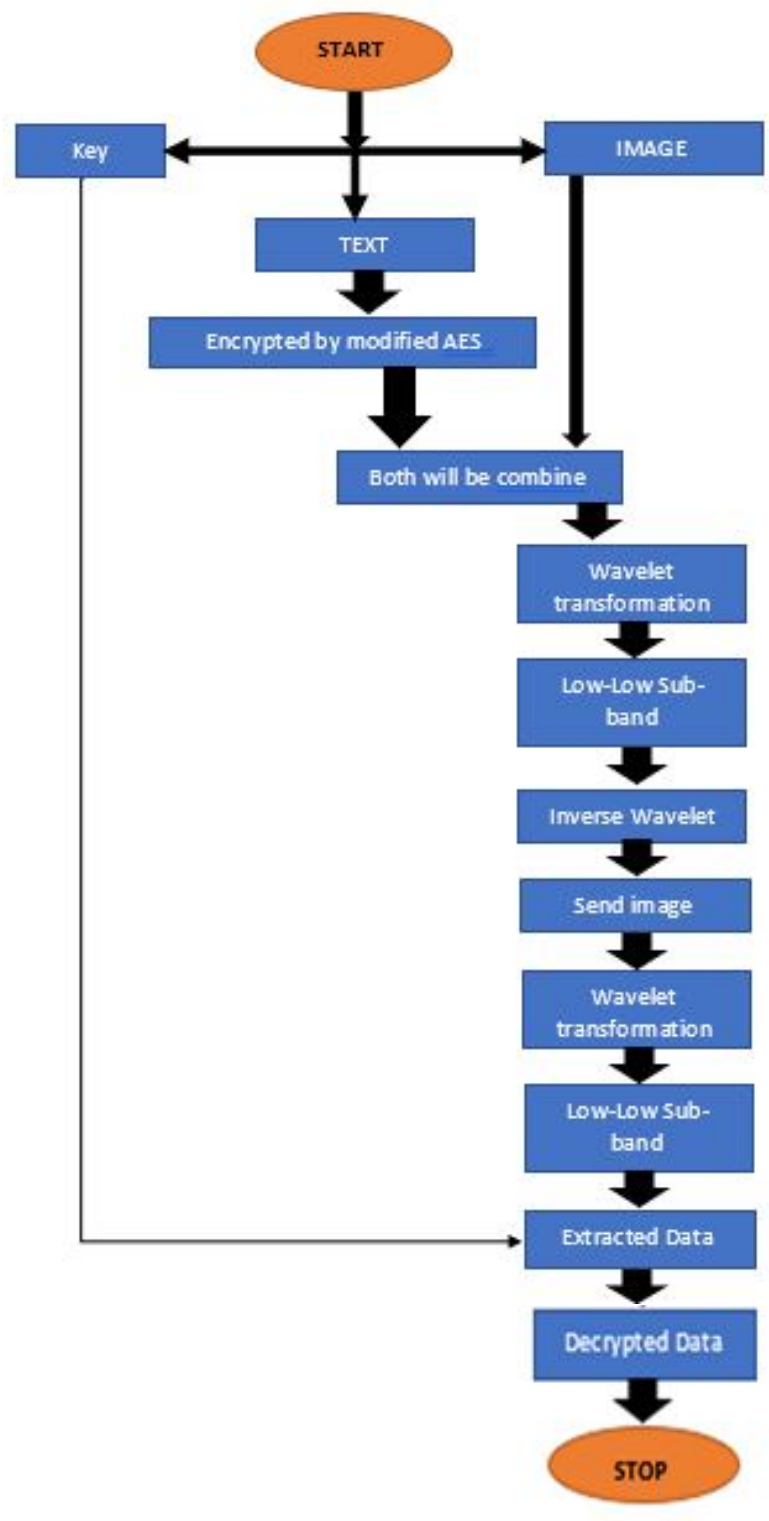

Figure 6: Propose Work Diagram

\section{RESULTS AND DISCUSSION}

By taking a model book record containing data ,Suraj-Kr.$\mathbf{1 9 m 4 2 8 m " , ~ t h e ~ l e n g t h ~ o f ~} 18$ character is taken to change the messages and taken a message to be hidden. This information will be implanted in a picture. Opposite end, the dataset picture changed utilizing Haar forward wavelet change to get Low-Low, Low-High, High-Low, and High-High subband. Information coming because of strategy inserted into subband 
(LL). From the point forward, a picture changed back to a structure utilizing Haar converse wavelet change. Subsequent to accepting the picture from the sender, the picture by and by gets changed utilizing Haar forward wavelet to extricate shrouded information. Information decoded utilizing means given in after images. All these data encrypted and decrypted using the steps shown in Figures 7 to Figures 12. At last, a message is gotten as ,Suraj-Kr.- 19m428m".

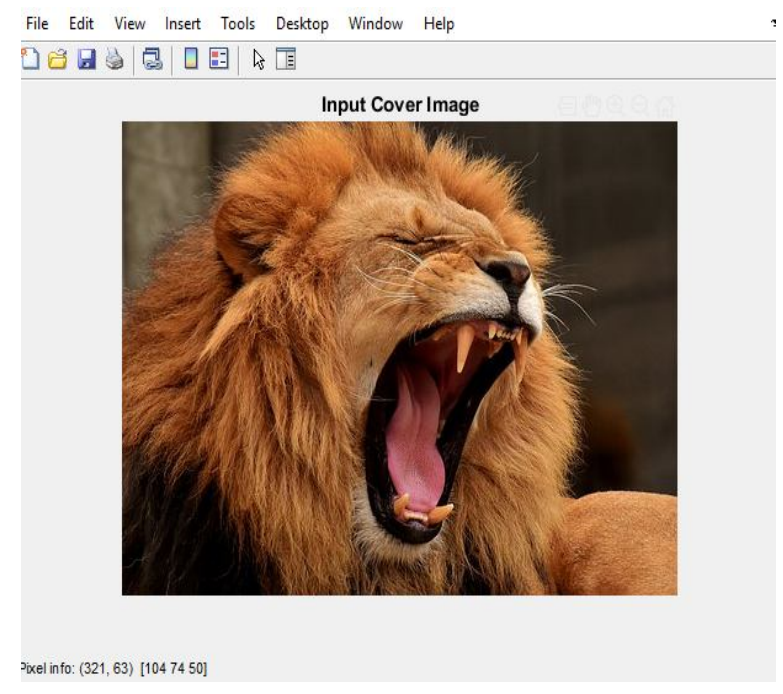

Figure 7: Original image

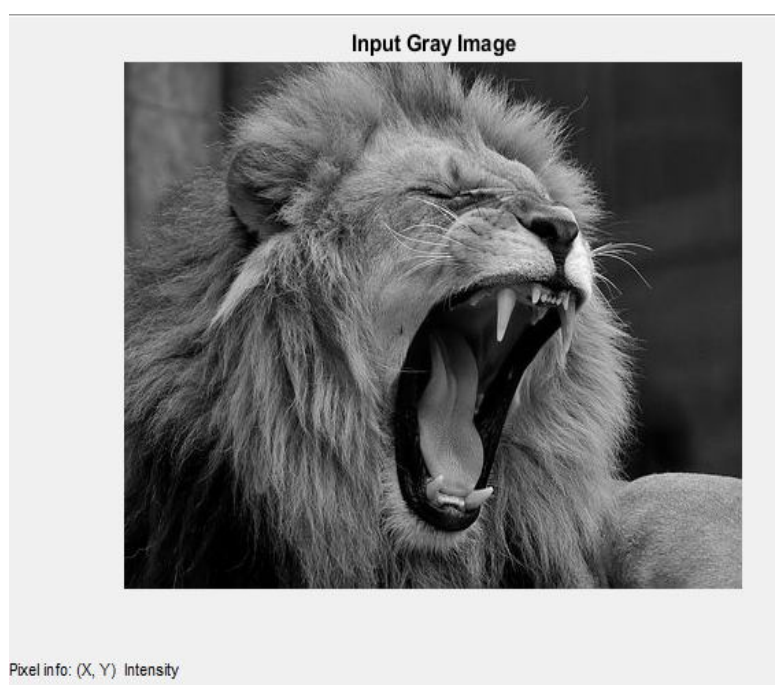

Figure 8: rgb to gray

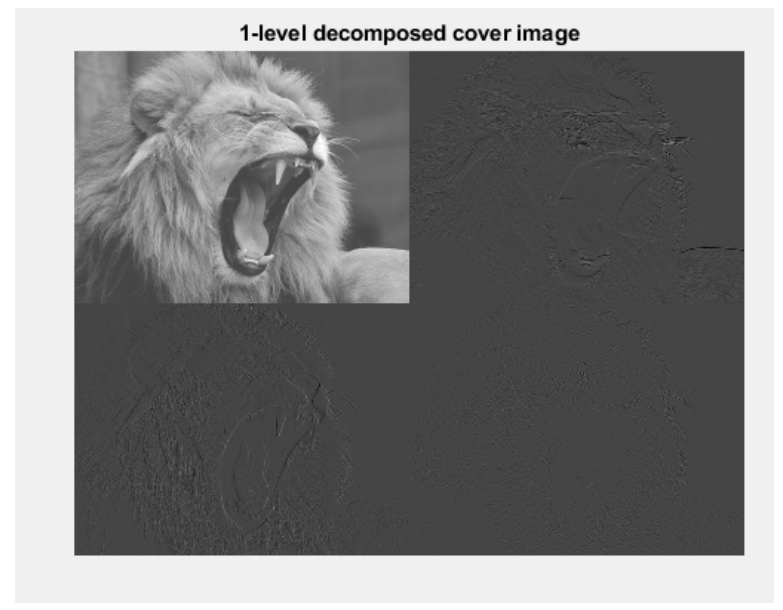

Figure 9: Decomposed level-1

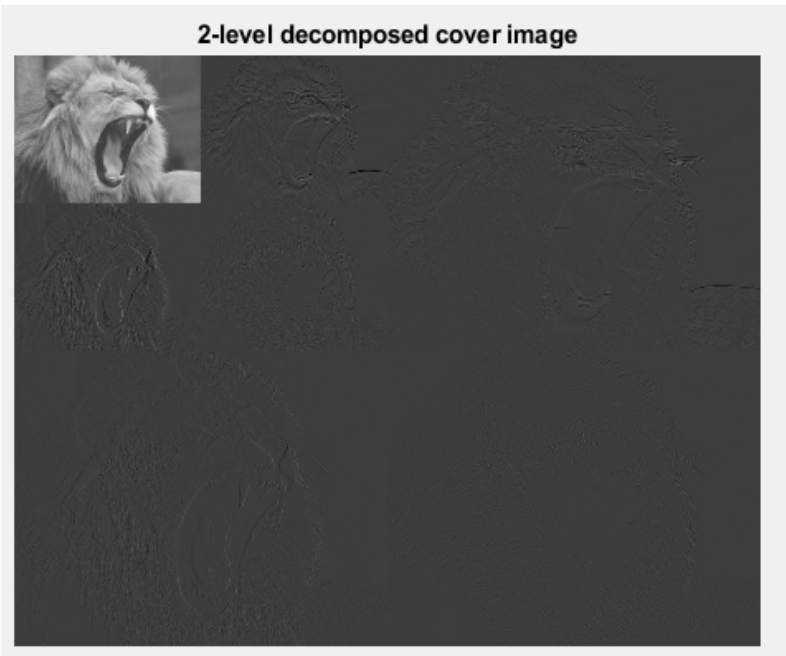

Figure 10: Decomposed level-2

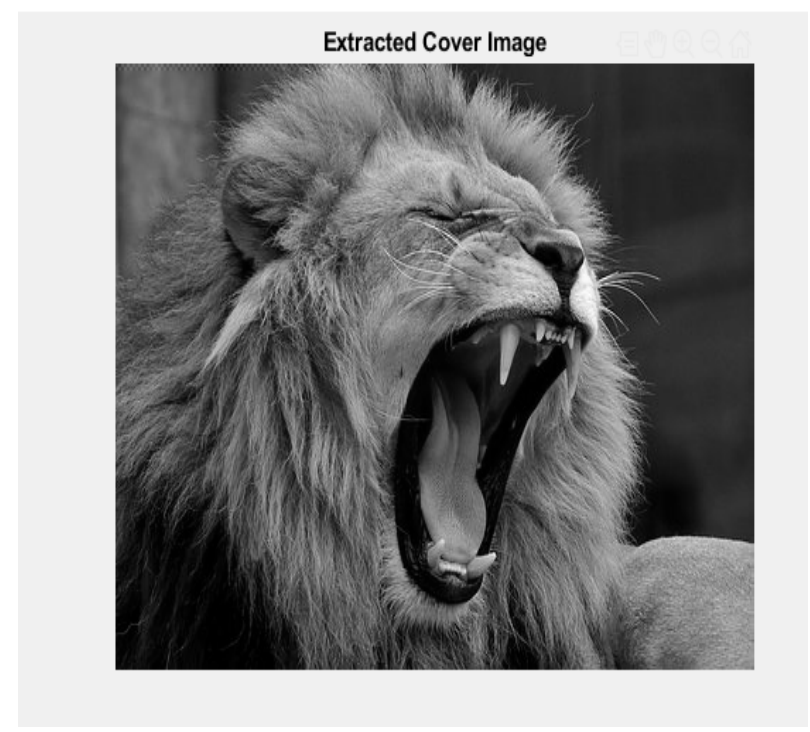

Figure 11: Extracted image 


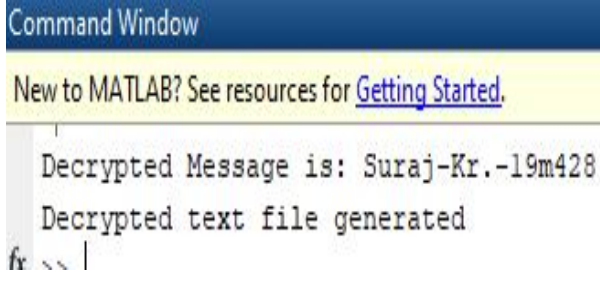

Figure 12: Decrypt Message

\section{CONCLUSION}

Cryptographic calculation alone certainly a not safe method to utilizing for information transmissions. Another strategy combines of cryptography and steganography given to give good choice for information transmissions. In this undertaking technique to join steganography (LSM) and cryptography (AES) is considered, to give a safer method to information transmissions through unstable and public organizations. Additional expansion securities of information, an encoded text isn't installed in a picture itself. It is installed in sub-band (LL) of wavelets changed a picture.

\section{REFERENCES}

1. Swanson, M., kobayashi, M., \& Tewfik, A. (1998). Multimedia Data-Embedding and Watermarking Technologies. IEEE Xplore, 1064-1087.

2. Thien, C.-C., \& Lin, J.-C. (2003). A simple andhigh-hiding capacity methodfor hiding. Pattern Recognition, 2875-2881.

3. Wang, R.-Z., Lin, C.-F., \& Lin, J.-C. (November 1999). Image hiding by optimal LSB substitution. Pattern Recognition(34), 671-683.

4. V.Kartalopoulos, S. (2009). Security of Information and Communication Networks. Hoboken,New Jersey: A john Wiley \& Sons,Inc.,.

5. Zeng, X.-t., Li, Z., \& Ping, L.-d. (2012). Reversible data hiding scheme using reference pixel and multi-layer embedding. International Journal of Electronics and Communications(66), 532-539.

6. Jung, K.-H., \& Yoo, \&.-Y. (2014). Steganographic method based on interpolation and LSB. Multimedia tools and applications, 2143-2155.

7. Chang, C.-K., \& Cheng, L. (2004). Hiding data in images by simple LSB substitution. Pattern Recognition, 469-474.

8. Mielikainen, J. (May 2006). LSB Matching Revisited. IEEE SIGNAL PROCESSING LETTERS, 12, pp. 285-287.

9. Kumar, V. S., \& Reddy, M. S. (2012). Image Compression Techniques by using Wavelet Transform. Information Engineering and Applications, 35-39.

10. Msolli, A., Helali, A., \& Maaref, H. (2018). New security approach in real-time wireless multimedia sensor networks. Computers \& Electrical Engineering, 72, 910-925.

11. LIAW, J.-J., CHANG, L.-H., \& LIAO, Y.-S. (2007). An Improvement of Robust and Blind Data Hiding Based on Self. WSEAS International Conference on Computer Engineering and Applications, (pp. 259-263). Gold Coast, Australia.

12. Radhakrishnan, R., Kharrazi, M., \& Memon, N. (2005). Data Masking: A New Approach for Steganography? VLSI signal processing systems for signal, image and video technology (41), 293-303. 